\title{
Detection of amplitude modulation with squeezed light for sensitivity beyond the shot-noise limit
}

\author{
Min Xiao, Ling-An Wu, and H. J. Kimble \\ Department of Physics, University of Texas at Austin, Austin, Texas 78712
}

Received January 22, 1988; accepted March 21, 1988

\begin{abstract}
An improvement in precision beyond the limit set by the vacuum-state or zero-point fluctuations of the electromagnetic field is reported for the detection of amplitude modulation encoded on a weak signal beam. The improvement is achieved by employing the squeezed light from an optical parametric oscillator to reduce the level of fluctuations below the shot-noise limit. An increase in signal-to-noise ratio of $2.5 \mathrm{~dB}$ relative to the shot-noise limit is demonstrated.
\end{abstract}

An exploration of the limits on the detection of small changes in amplitude of the electromagnetic field is of fundamental and practical importance in optical physics. While a variety of noise sources of technical origin often limit sensitivity, the fundamental limit on the detection of a small absorption or loss $\gamma$ has been the so-called shot-noise limit (SNL), which sets a lower bound on the minimum detectable change $\delta A$ of the coherent amplitude $A$ of the field given by $\gamma \cong \delta A / A \cong$ $1 / \sqrt{N}$, where $N$ is the number of photons recorded in the time interval of the measurement. A diverse set of experiments in ultrasensitive laser spectroscopy ${ }^{1}$ provides but one example in which it has been possible to overcome technical noise, to eliminate extraneous coherent signals, and to reach the SNL. However, with conventional laser light in a coherent state, it is not possible to proceed further to a sensitivity greater than that specified by the SNL.

Improvements in precision beyond the SNL can be realized by employing squeezed states of light. A squeezed state is characterized by a phase-dependent redistribution of quantum fluctuations such that the variance of one of two quadrature components of the field is reduced below that of the vacuum state. ${ }^{2}$ By encoding the signal (e.g., amplitude modulation) on the field variable with reduced fluctuations and by employing a detection scheme that is largely insensitive to the increased fluctuations of the conjugate field variable, sensitivity greater than the SNL can be achieved. ${ }^{3,4}$

In this Letter we report experiments that utilize squeezed light to improve the sensitivity for the detection of amplitude modulation by $2.5 \mathrm{~dB}$ relative to the shot-noise or vacuum-state limit. In the measurements a weak signal beam is combined with either a vacuum-state or a squeezed-state input; the amplitude of the resulting beam is modulated in propagation through an acousto-optic modulator. The observed increase in sensitivity for the detection of the signal modulation is currently limited principally by simple linear losses in propagation and detection and not by the degree of squeezing available from our source. ${ }^{5,6}$

Our apparatus is depicted in general outline in Fig. 1. Consider first the case of the inputs to $m_{1}$ being a coherent state $\hat{E}_{0}$ and a vacuum state $\hat{E}_{s}$, with the transmissivity of $m_{1}$ being small. ${ }^{7}$ The weak probe beam $\hat{E}_{1}$ that is output from $\mathrm{m}_{1}$ is modulated in amplitude at frequency $\Omega_{0}$ by an acousto-optic modulator $(\mathrm{AOM})$ to generate a field $\hat{E}_{2}$ given by ${ }^{8}$

$$
\left\langle\hat{E}_{2}\right\rangle=\left\langle\hat{E}_{1}\right\rangle \sqrt{T} \cos \theta(t)
$$

where $\theta(t)=\theta_{0}+\theta_{1} \cos \Omega_{0} t$ and is proportional to the amplitude of the index of refraction variation produced by the traveling acoustic wave in the modulator. $T$ is the static transmission coefficient $(\theta=0)$ of the AOM. For the case of low-loss operation of the modulator we expand Eq. (1), keeping only lowest-order terms at the modulation frequency $\Omega_{0}$ to find that

$$
\left\langle\hat{E}_{2}\right\rangle \cong\left\langle\hat{E}_{1}\right\rangle T\left(1-\delta \cos \Omega_{0} t\right),
$$

where the modulation index $\delta \equiv \theta_{1} \sin \theta_{0}$. The modulated field $\hat{E}_{2}$ enters the signal port of a balanced homodyne detector formed by the beam splitter $\mathrm{m}_{2}$

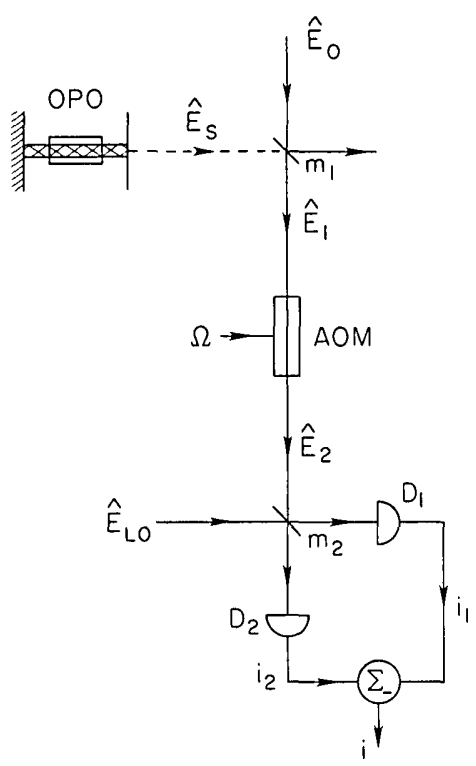

Fig. 1. Schematic of the apparatus for detection of amplitude modulation with squeezed states. The reflectivity of $\mathrm{m}_{1}$ is close to unity; $\mathrm{m}_{2}$ is a beam splitter with $R=T=0.5$. 
(50-50) and the photodiodes $\mathrm{D}_{1}$ and $\mathrm{D}_{2}$ and operated such that $\left|\left\langle\hat{E}_{\mathrm{LO}}\right\rangle\right| \gg\left|\left\langle\hat{E}_{2}\right\rangle\right| .{ }^{9}$ The amplified photocurrents $i_{1}$ and $i_{2}$ from the two arms are combined with a $180^{\circ}$ phase shift to produce the photocurrent $i$, which is directed to a commercial spectrum analyzer.

The photocurrent $i$ contains a signal component $i_{s}$ at frequency $\Omega_{0}$ of rms amplitude

$$
i_{s}\left(\Omega_{0}\right)=\sqrt{2 T} e \alpha \eta\left\langle\hat{E}_{\mathrm{LO}}\right\rangle\left\langle\hat{E}_{1}\right\rangle \delta \cos \beta,
$$

where the units of the fields are chosen such that $\left\langle\hat{E}^{\dagger} \hat{E}\right\rangle$ expresses a flux in photons per second across the detector area. The quantum efficiency of the photodiodes is given by $\alpha$, while $\eta$ expresses the heterodyne efficiency for overlap of the fields $\hat{E}_{2}$ and $\hat{E}_{\mathrm{LO}}$. $\beta$ is the phase between $\left\langle\hat{E}_{2}\right\rangle$ and $\left\langle\hat{E}_{\mathrm{LO}}\right\rangle$. For $\beta=\beta_{+}=$ $p \pi$ ( $p$ an integer), the fields are in phase, and the amplitude modulation encoded on $\hat{E}_{2}$ results in amplitude modulation of $\hat{E}_{\mathrm{LO}}$. For $\beta=\beta_{-}=(2 p+1) \pi / 2$, the modulation of $\hat{E}_{2}$ results in phase modulation of $\hat{E}_{\mathrm{LO}}$ and hence produces no signal current to lowest order in $\delta$. In addition to the signal current $i_{s}\left(\Omega_{0}\right)$, there is a noise current $i_{n}$ against which any coherent detection must be made. In the case of a vacuumstate input for $\hat{E}_{s}, i_{n}$ is just the shot-noise current of rms amplitude

$$
i_{n}=(2 e i B)^{1 / 2}=\left(2 e^{2} \alpha\left\langle\hat{E}_{\mathrm{LO}}^{\dagger} \hat{E}_{\mathrm{LO}}\right\rangle B\right)^{1 / 2} .
$$

The signal-to-noise ratio for coherent-state inputs $\left(\hat{E}_{0}\right.$, $\left.\hat{E}_{\mathrm{LO}}\right)$ and a vacuum-state input $\hat{E}_{s}$ is thus

$$
\Psi_{v} \equiv \frac{i_{s}^{2}}{i_{n}^{2}}=\frac{\alpha P_{2} \cos ^{2} \beta}{B} \delta^{2},
$$

where we have defined the effective power $P_{2} \equiv$ $T \eta^{2}\left|\left\langle\hat{E}_{1}\right\rangle\right|^{2}$ (photons/sec). For $\beta=\beta_{+}$, a signal-tonoise ratio of unity $\left(\Psi_{v}=1\right)$ implies a minimum detectable modulation index $\delta=\left(B / \alpha P_{2}\right)^{1 / 2}=1 / \sqrt{N_{2}}$, with $N_{2}$ as the mean number of photoelectrons in the measurement interval $B^{-1}$ due to $P_{2}$. This limit on the minimum detectable amplitude modulation is the SNL discussed above with the association of $\delta$ with $\gamma$. Any improvement beyond this limit is not possible in conventional detection schemes with coherent states.

An improvement in sensitivity is possible, however, if a squeezed field $\hat{E}_{s}$ is injected in place of the vacuum field at $m_{1}$ to reduce the noise against which the modulation signal must be detected. The squeezed light in our experiment is generated by a subthreshold optical parametric oscillator with degenerate signal and idler waves at $\lambda=1.064 \mu \mathrm{m} .5,6$ The output field $\hat{E}_{s}$ from the optical parametric oscillator is characterized by a spectrum of squeezing $S(\Omega, \phi)$, where $\Omega$ is the frequency offset from the carrier and $\phi$ selects the quadrature phase. ${ }^{10} S(\Omega, \phi)$ is normalized such that $S=0$ for a vacuum state, while for a perfectly squeezed field, $S \rightarrow$ -1 for one quadrature and $S \rightarrow \infty$ for the conjugate quadrature. With the injection of squeezed vacuum at $m_{1}$ and under the assumptions of modest squeezing and small modulation index, the calculation of the signal current proceeds exactly as before [Eq. (3)]. However, the noise in the difference photocurrent now becomes $i_{n}^{2}(\Omega, \phi)=i_{n}^{2}[1+\zeta S(\Omega, \phi)]$, resulting in a signal-to-noise ratio

$$
\Psi\left(\Omega_{0}, \phi\right)=\Psi_{v} /\left[1+\zeta S\left(\Omega_{0}, \phi\right)\right]=\frac{\alpha P_{2} \delta^{2} \cos ^{2} \beta}{B\left[1+\zeta S\left(\Omega_{0}, \phi\right)\right]},
$$

where $\zeta$ represents the overall efficiency for propagation and detection of the squeezed light. ${ }^{6}$ The two phases $(\beta, \phi)$ appearing in Eq. (6) specify, respectively, the orientation of the coherent modulation at $\Omega_{0}$ and the orientation of the squeezed-noise ellipse relative to the local-oscillator field. For $\phi=\phi_{+}=q \pi$ ( $q$ an integer), the enhanced fluctuations $S_{+}$of the squeezed state align with $\left\langle\hat{E}_{\mathrm{LO}}\right\rangle$, while for $\phi=\phi_{-}=(2 q+1) \pi / 2$ the reduced fluctuations $S_{-}$of the squeezed state align with $\left\langle E_{\mathrm{LO}}\right\rangle$. From Eq. (6) we see that for $\phi=\phi_{-}$, an improvement in signal-to-noise ratio beyond the shotnoise or vacuum-state limit is given by the factor $(1+$ $\left.\zeta S_{-}\right)^{-1} \gg 1$ for $S_{-} \rightarrow-1$ and $\zeta \simeq 1$. On the other hand, for $\phi=\phi_{+}$, there is a large degradation in the signal-tonoise ratio by the factor $\left(1+\zeta S_{+}\right)^{-1} \ll 1$, pointing out the requirement for precise control of $\phi$.
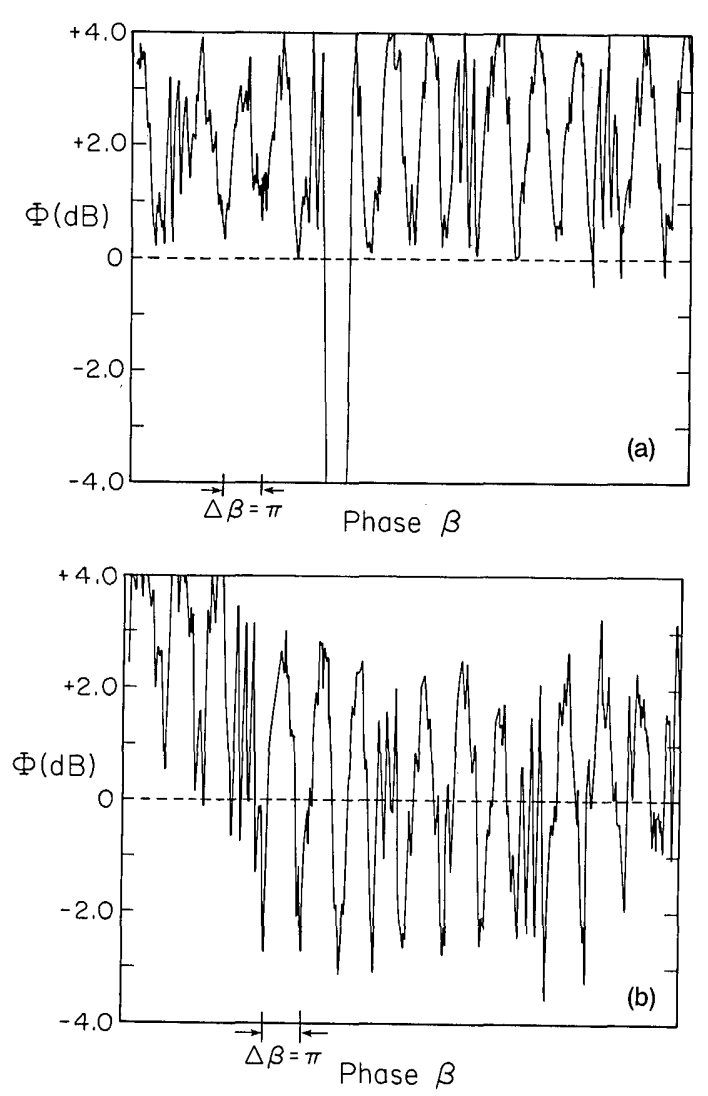

Fig. 2. Spectral density $\Phi$ of photocurrent fluctuations versus signal phase $\beta$ for fixed analysis frequency $\Omega_{0} / 2 \pi=1.6$ $\mathrm{MHz}$. The phase $\beta$ between $\left\langle E_{2}\right\rangle$ and $\left\langle E_{\mathrm{LO}}\right\rangle$ is swept in a sawtooth fashion to produce the periodic variation in $\Phi$ with the sharp features corresponding to the flyback to restart the scan. The dashed line gives the vacuum level obtained with inputs $E_{0}$ and $E_{s}$ blocked. The gap in (a) is caused by a trigger event that interrupts the local-oscillator beam. (a) Vacuum-state input for $E_{s}$, (b) squeezed-state input for $E_{s}$. The slow variation of $\Phi$ over the scan in (b) results from a variation in $\phi$ to adjust for minimum noise level. An improvement of $2.5 \mathrm{~dB}$ in signal-to-noise ratio is achieved in (b) relative to (a) near the noise minimum. The time for the entire trace is $0.2 \mathrm{sec}$. 
Our experimental results for the improvement in sensitivity for the detection of amplitude modulation are displayed in Fig. 2, which records the spectral density $\Phi$ of photocurrent fluctuations of the difference photocurrent $i$ as a function of phase $\beta$. The periodic up-down variation of $\Phi$ with $\beta$ reflects the $\cos \beta$ dependence of the signal photocurrent as given by Eq. (3). For $\beta=\beta_{+}$, the signal modulation is detected with maximum efficiency $\left(\cos ^{2} \beta=1\right)$, and the detected level $\Phi^{\prime}$ is the sum of contributions from the coherent modulation and the noise current. For $\beta=\beta_{-}$, the modulation is predominantly frequency modulation with respect to the local-oscillator field, and no coherent signal is expected $\left(\cos ^{2} \beta=0\right)$. Hence the detected level in this situation should correspond to photocurrent noise alone, as given by $\Phi=10 \log (1+\zeta S)$. In Fig. 2(a) the input $\hat{E}_{s}$ to $m_{1}$ is a vacuum state for which $S=0$, while in Fig. 2(b) a squeezed state is injected for which $S<0$ for $\phi$ properly chosen. The actual vacuum-state or shot-noise level in Fig. 2 is indicated by a dashed line and is obtained with the inputs $\hat{E}_{s}$ and $\hat{E}_{0}$ blocked after the procedure discussed in Ref. 6. Figure 2(a) corresponds roughly to the case $\Psi \simeq 1$ (signalto-noise ratio of unity) and represents the SNL for signal detection. A decrease in noise level and an increase in signal-to-noise ratio beyond this limit are shown in Fig. 2(b). Figures 2(a) and 2(b) are recorded under identical conditions with the single exception that squeezed-vacuum light is injected into $\mathrm{m}_{1}$ for Fig. 2(b). In Fig. 2(b) the angle $\phi$ is slowly varied to reach a position of minimum noise. On the left-hand edge of the figure the noise level is well in excess of the SNL. As a value near $\phi=\phi_{-}$is reached in the center of the figure, there is a decrease in photocurrent noise below the SNL. From a number of traces as in Fig. 2, we find an improvement in signal-to-noise ratio relative to the vacuum-state limit of $(2.5 \pm 0.5) \mathrm{dB}$ (a factor of 1.8 in terms of noise power). The consequence of this improvement is that a given level of precision for $i_{s}\left(\Omega_{0}\right)$ could be reached in a measurement time shortened by a factor $\sqrt{1.8}$ relative to shot-noise-limited measurements. Alternatively, for a fixed measurement interval, sensitivity for signal detection is enhanced by the factor $\sqrt{1.8}$

The experimental conditions relevant to the data of Fig. 2 are as follows. The incident local-oscillator power is $6.4 \times 10^{-4} \mathrm{~W}$, the detector quantum efficiencies $\alpha \cong 0.89$, and the shot noise is in excess of amplifier noise by $20 \mathrm{~dB}$ at the modulation frequency $\Omega_{0} / 2 \pi=$ 1.6 MHz. The power $P_{2}=8 \times 10^{-8} \mathrm{~W}$, while $\delta=8.5 \times$ $10^{-4}, \theta_{1} / \sin \theta_{0}=0.015$, and $T>0.98$. The detection bandwidth is $1.0 \times 10^{5} / \mathrm{sec}$ (Gaussian filter) with two postdetection video filters of time constants $\left(10^{-4} \mathrm{sec}\right.$, $\left.5 \times 10^{-4} \mathrm{sec}\right)$. The signal-to-noise ratio evidenced in Fig. 2(a) allows us to infer a modulation index $\delta^{\prime}=8.5$ $\times 10^{-4}$, in excellent agreement with the measured modulation index $\delta$.

We should note that for the particular arrangement that we have employed, the sensitivity could be greatly enhanced by simply removing $\mathrm{m}_{1}$ to increase the field $\left\langle\hat{E}_{1}\right\rangle$ and hence the amplitude of the amplitude-modulation sidebands. Indeed, the case of squeezed light in the configuration of Fig. 1 can only improve the sensitivity to the level associated with $\left\langle\hat{E}_{0}\right\rangle$ alone. However, there are many applications (especially with regard to spectroscopy) for which there are natural upper limits to the fields that can be used to illuminate various samples and for which the incident laser is attenuated to provide a probe beam precisely in the fashion of $m_{1}$. If such attenuation is not permitted, the squeezed vacuum beam $\hat{E}_{s}$ and the coherent field $\hat{E}_{0}$ can be combined not at a simple mirror but rather with an optical cavity. The linewidth $\kappa$ of the cavity would be chosen to be small compared with that associated with $S(\Omega)$. With the cavity locked at resonance, the fields would be combined with $\hat{E}_{0}$ transmitted through the cavity and $\hat{E}_{s}$ reflected from the cavity output mirror. A squeezed coherent beam of approximate amplitude $\left\langle\hat{E}_{0}\right\rangle$ with a spectrum of squeezing given by $S(\Omega)$ could then be achieved, except for a loss of squeezing around $\Omega=0$ of width $\kappa$. That the optical parametric oscillator below threshold produces a squeezed vaccum state and not a squeezed coherent state is thus of little consequence.

In conclusion, we have demonstrated an improvement in sensitivity of $2.5 \mathrm{~dB}$ for the measurement of amplitude modulation beyond the limit set by the vacuum-state or zero-point fluctuations of the electromagnetic field. Although the arrangement employed may seem somewhat artificial relative to an actual spectroscopic application, we note that the replacement of the AOM by a sample whose absorption is externally modulated at $\Omega_{0}$ would provide a workable squeezed-state spectrometer. In addition, the measurement that we have described can be transcribed into the language of frequency-modulation spectroscopy by the simple association $\theta_{1} \sin \theta_{0}=M \mu$, where $M$ is the modulation index of the frequency-modulated sidebands and $\mu$ the differential sideband absorption. ${ }^{1}$

We gratefully acknowledge the contributions of J. L. Hall. This research was supported by the U.S. Office of Naval Research and by the Venture Research Unit of British Petroleum America.

\section{References}

1. J. S. Snyder and R. A. Keller, eds., Ultrasensitive Laser Spectroscopy, J. Opt. Soc. Am. B 2, 1428-1593 (1985).

2. H. J. Kimble and D. F. Walls, eds., Squeezed States of the Electromagnetic Field, J. Opt. Soc. Am. B 4, 14501741 (1987).

3. M. Xiao, L.-A. Wu, and H. J. Kimble, Phys. Rev. Lett. 59, 278 (1987).

4. P. Grangier, R. E. Slusher, B. Yurke, and A. LaPorta, Phys. Rev. Lett. 59, 2153 (1987).

5. L.-A. Wu, H. J. Kimble, J. L. Hall, and H. Wu, Phys. Rev. Lett. 57, 2520 (1986).

6. L.-A. Wu, M. Xiao, and H. J. Kimble, J. Opt. Soc. Am. B 4, 1465 (1987).

7. We denote Hilbert-space operators with a caret $\left({ }^{\wedge}\right)$.

8. A. Yariv, Optical Electronics (Holt, Rinehart, and Winston, New York, 1985), Chap. 12.

9. H. P. Yuen and V. W. S. Chan, Opt. Lett. 8, 177 (1983).

10. M. J. Collett and C. W. Gardiner, Phys. Rev. A 30, 1386 (1984). 